\title{
Performance of diffusion and perfusion MRI in evaluating primary central nervous system lymphomas of different locations
}

\author{
Zhen Xing ${ }^{1}$, Nannan Kang ${ }^{2}$, Yu Lin², Xiaofang Zhou', Zebin Xiao ${ }^{3}$ and Dairong Cao ${ }^{1 *}$ (D)
}

\begin{abstract}
Background: Diffusion and perfusion MRI can invasively define physical properties and angiogenic features of tumors, and guide the individual treatment. The purpose of this study was to investigate whether the diffusion and perfusion MRI parameters of primary central nervous system lymphomas (PCNSLS) are related to the tumor locations.

Methods: We retrospectively reviewed the diffusion, perfusion, and conventional MRI of 68 patients with PCNSLs at different locations (group 1: cortical gray matter, group 2: white matter, group 3: deep gray matter). Relative maximum cerebral blood volume ( $\mathrm{rCBV}_{\max }$ ) from perfusion $\mathrm{MRI}$, minimum apparent diffusion coefficients ( $\mathrm{ADC} \mathrm{C}_{\min }$ ) from DWI of each group were calculated and compared by one-way ANOVA test. In addition, we compared the mean apparent diffusion coefficients $\left(A D C_{\text {mean }}\right)$ in three different regions of control group.

Results: The rCBV $\max$ of PCNSLs yielded the lowest value in the white matter group, and the highest value in the cortical gray matter group $(P<0.001)$. However, the $A D C_{\text {min }}$ of each subgroup was not statistically different. The $A D C_{\text {mean }}$ of each subgroup in control group was not statistically different.

Conclusion: Our study confirms that rCBV $_{\max }$ of PCNSLs are related to the tumor location, and provide simple but effective information for guiding the clinical practice of PCNSLS.
\end{abstract}

Keywords: Primary central nervous system lymphomas, diffusion, Perfusion

\section{Background}

Primary central nervous lymphomas (PCNSLs) are malignant tumors with increased incidence rates [1]. To date, methotrexate-based chemotherapy is the cornerstone for PCNSLs treatment. The response to initial high-dose chemotherapy is a reliable indicator of the patients' long-term survival $[2-5]$. As proposed by a previous study, the chemotherapy response is predominantly determined by the delivery of drugs through the vascular system [6]. Besides, some researchers have documented

\footnotetext{
* Correspondence: dairongcao@163.com

'Department of Radiology, First Affiliated Hospital of Fujian Medical University, 20 Cha-Zhong Road, Fuzhou 350005, Fujian, China

Full list of author information is available at the end of the article
}

that PCNSLs with different cellular growth patterns may have different prognoses $[7,8]$.

Diffusion-weighted imaging (DWI) can assess the Brownian movement of water in the microscopic tissue environment by using apparent diffusion coefficient (ADC) values. Densely packed tumor cells with a high nuclear-to-cytoplasmic ratio could reduce water molecule motion $[9,10]$. Few studies have suggested a significant correlation between ADC values and prognosis of patients with PCNSLs treated with methotrexate [11-13].

Dynamic susceptibility contrast perfusion-weighted imaging (DSC-PWI) can provide physiological information about vascular endothelial proliferation and 
angiogenesis [14-17]. Since tumor aggressiveness correlates with neovascularization, DSC-PWI is useful in the preoperative evaluation of brain tumors (including PCNSLs). Regional CBV (rCBV) derived from DSC-PWI can provide important hemodynamic information by intravenous injection of gadolinium-based contrast agent [18-20]. The response to chemotherapy is mainly determined by the delivery of drugs to the tumor through the vascular system [6]. Therefore, the rCBV value might be used as a biomarker of treatment responses in PCNSLs.

From the imaging perspective, diffusion and perfusion MRI may be helpful for a comprehensive assessment of the cellularity and vascularity, and a prediction of treatment response of PCNSLs. To our knowledge, there is no study in the literature combining diffusion and perfusion techniques to evaluate the PCNSLs of different locations. Therefore, our research aims to investigate whether DWI and DSC-PWI parameters are correlated with tumor locations, and provide evidence for clinical decision-making and prognostic evaluation.

\section{Methods}

\section{Patients}

The institutional review board of our hospital approved this study and informed consent was waived due to the retrospective design. Our institution's database identified 166 patients who underwent MR examination for potential PCNSLs between October 2009 and September 2017. The inclusion criteria were as follow: (a) immunocompetent patients, (b) histopathological diagnosis of PCNSLs, (c) available pretreatment conventional MRI (cMRI), DWI and DSC-PWI. Patients with poor image quality were excluded. Ultimately, 68 patients (31 males and 37 females, age range $29-78$ years; mean age 56.80 years) were included.

\section{MR imaging techniques}

All MR images were acquired in the routine clinical workup using a 3.0 Tesla MRI system (Magnetom Verio TIM; Siemens Healthcare, Erlangen, Germany) with an eight-channel head coil. The cMRI protocols consisted
Table 2 Comparison of DWI and DSC-PWI variables among the three groups of PCNSLs, and DWI variables among the three groups of control group

\begin{tabular}{lllll}
\hline & $\mathrm{CGM}$ & $\mathrm{WM}$ & $\mathrm{DGM}$ & $P$ \\
\hline $\mathrm{ADC}_{\min }$ & $0.61 \pm 0.15$ & $0.65 \pm 0.15$ & $0.57 \pm 0.14$ & .169 \\
$\left(10^{-3} \mathrm{~mm}^{2} / \mathrm{s}\right)$ & & & & \\
$\mathrm{ADC}_{\text {mean }}$ & $0.70 \pm 0.03$ & $0.69 \pm 0.03$ & $0.69 \pm 0.02$ & .202 \\
$\left(10^{-3} \mathrm{~mm}^{2} / \mathrm{s}\right)$ & & & & \\
$\mathrm{rCBV}{ }_{\max }$ & $2.55 \pm 0.64$ & $1.34 \pm 0.46$ & $1.87 \pm 0.74$ & $<.001$ \\
\hline
\end{tabular}

CGM Cortical gray matter; WM White matter; DGM Deep gray matter

of axial T1WI (TR/TE, $250 / 2.48 \mathrm{~ms}$ ), axial T2WI (TR/ TE, $4000 / 96 \mathrm{~ms}$ ), axial FLAIR (TR, $9000 \mathrm{~ms}$; TE, $94 \mathrm{~ms}$; TI, $2500 \mathrm{~ms}$ ), and contrast-enhanced T1WI (CE-T1WI; $\mathrm{TR} / \mathrm{TE}, 250 \mathrm{~ms} / 2.48 \mathrm{~ms}$ ) in 3 orthogonal planes. It was uniform in all series about FOV $220 \times 220 \mathrm{~mm}$, section thickness $5 \mathrm{~mm}$, and intersection gap $1.0 \mathrm{~mm}$.

DWI was performed in the axial plane with a spinecho-planar sequence (TR/TE, 8200/102 ms; NEX, 2.0; b-values, 0 and $1000 \mathrm{~s} / \mathrm{mm} 2$ ). Corresponding ADC maps were generated automatically by the MRI system.

DSC-PWI was achieved with a gradient-recalled T2*weighted echo-planar imaging sequence (TR/TE, 10001250/54 ms; NEX, 1.0; flip angle, $35^{\circ}$ ). In the first three phases, non-enhanced images were scanned to establish a pre-contrast baseline. When the scan was to the fourth phase of DSC-PWI, a standard dose of $0.1 \mathrm{mmol} / \mathrm{kg}$ of gadobente dimeglumine (Gd-BOPTA) was injected intravenously with a flow rate of $3 \mathrm{ml} / \mathrm{s}$, followed by a $20 \mathrm{ml}$ continuous saline flush.

\section{Data processing}

All imaging assessments were performed on a Siemens workstation with standard software. All cMRI data concerning notch sign and contrast-enhancement pattern were assessed by two neuroradiologists who were blinded to tumor histology. When two observers disagreed, a senior neuroradiologist made the final decision.

To assess DWI data, ADC values were measured by manually placing ROIs on the ADC maps. At least five small round ROIs $\left(25-40 \mathrm{~mm}^{2}\right)$ were selected inside the

Table 1 The main clinical and CMRI features of three groups of PCNSLS

\begin{tabular}{|c|c|c|c|c|}
\hline & CGM & WM & DGM & P \\
\hline Sex (male/female) & $15 / 17$ & $7 / 15$ & $9 / 5$ & .159 \\
\hline Age (year) & $56.09 \pm 9.8$ & $57.96 \pm 12.53$ & $52.50 \pm 13.83$ & .393 \\
\hline Contrast-enhancement pattern & & & & .234 \\
\hline Homogeneous & 29 & 30 & 19 & \\
\hline Heterogeneous & 10 & 5 & 2 & \\
\hline Notch sign & & & & .657 \\
\hline Yes & 33 & 31 & 17 & \\
\hline No & 6 & 4 & 4 & \\
\hline
\end{tabular}

CGM Cortical gray matter; WM White matter; DGM Deep gray matter 
tumor areas of visually lowest ADC. The ROI placements were made from the enhancing solid portion of the lesion, avoiding necrotic, cystic, hemorrhagic, or visible blood vessel that might affect the ADC values. For each patient, the enhancing solid portion of the tumor was identified on T2WI and CE-T1WI. Finally, the minimum ADC $\left(A D C_{\text {min }}\right)$ was calculated from the ROI with the lowest $\mathrm{ADC}$ value. The mean $\mathrm{ADC}\left(\mathrm{ADC}_{\text {mean }}\right)$ values of normal cortical gray matter (CGM), white matter (WM), and deep gray matter (DGM) were also calculated. ADC values were expressed as $\times 10^{-3} \mathrm{~mm}^{2} / \mathrm{s}$.

To evaluate DSC-PWI data, whole-brain CBV maps were generated using a single-compartment model. The relative maximum $\mathrm{CBV}$ value $\left(\mathrm{rCBV}_{\max }\right)$ was calculated by dividing the maximum $\mathrm{CBV}$ value of the tumor by the mean $\mathrm{CBV}$ value of the contralateral unaffected white matter. Therefore, the rCBV value was used as a quantitative parameter without unit. To minimize variances in $\mathrm{rCBV}_{\max }$ values in each patient, measurements of $\mathrm{rCBV}_{\max }$ values were performed with the same ROIs as those used for ADC measurement.

All the parameters derived from DWI and DSC-PWI were measured by a neuroradiologist (Z.X. with 9 years of experience in brain imaging) who blinded to the tumor histology.

\section{Statistical analysis}

All quantitative parameters are presented as the means \pm standard deviation (SD). A one-way ANOVA test was performed for the $\mathrm{ADC}_{\text {min }}, \mathrm{ADC}_{\text {mean }}$ and $\mathrm{rCBV}_{\text {max }}$ values with a Least Significant Difference (LSD) among groups. All $P$-values $<0.05$ were considered to represent statistical significance. Statistical analysis was performed using SPSS software (Version 22.0, SPSS Inc., Chicago, USA) and MedCalc (Version 12.1.0, MedCalc Inc., Mariakierke, Belgium).

\section{Results}

There was a total of 68 patients with 95 lesions. Totally 73 lesions (single lesion in 36 cases and multiple lesions in 17 cases) underwent DSC-PWI and 82 lesions (single lesion in 37 cases and multiple lesions in 23 cases) underwent DWI. The clinical and cMRI characteristics were summarized in Table 1 . Homogenous contrastenhancement pattern and notch sign were non-specific observed in PCNSLs regardless of location.

Table 2 and Fig. 1 showed the results of $\mathrm{rCBV}_{\max }$, $\mathrm{ADC}_{\text {mean }}$ and $\mathrm{ADC}_{\text {min }}$ for the three groups. A significant difference of $\mathrm{rCBV}_{\max }$ was found among the three locations $(P<0.001)$. As shown in Figs. $1,2,3,4$, the $\mathrm{rCBV}_{\max }$ values were lowest in WM and highest in CGM. However, $\mathrm{ADC}_{\min }$ values of subgroup tumors

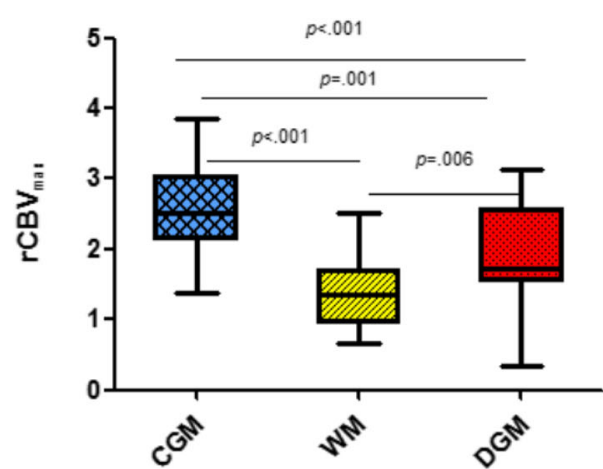

A

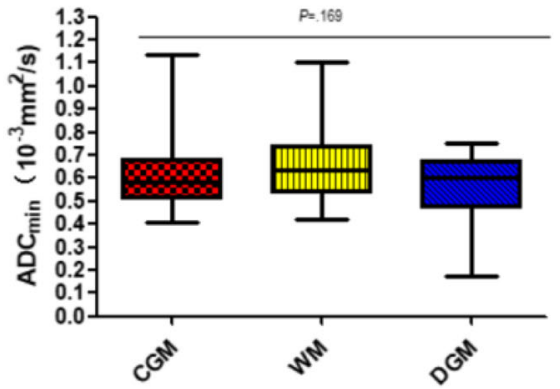

B

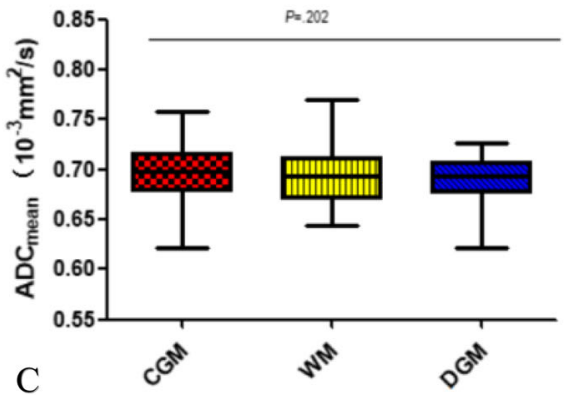

Fig. 1 Comparison of (a) relative maximum cerebral blood volume $\left(r C B V_{\max }\right)$ and $(\mathbf{b})$ minimum apparent diffusion coefficient $\left(A D C_{\min }\right)$ among PCNSLs of different locations of cortical gray matter (CGM), white matter (WM); deep gray matter (DGM), and (c) mean apparent diffusion coefficient $\left(A D C_{\text {mean }}\right)$ among control group of three different locations 


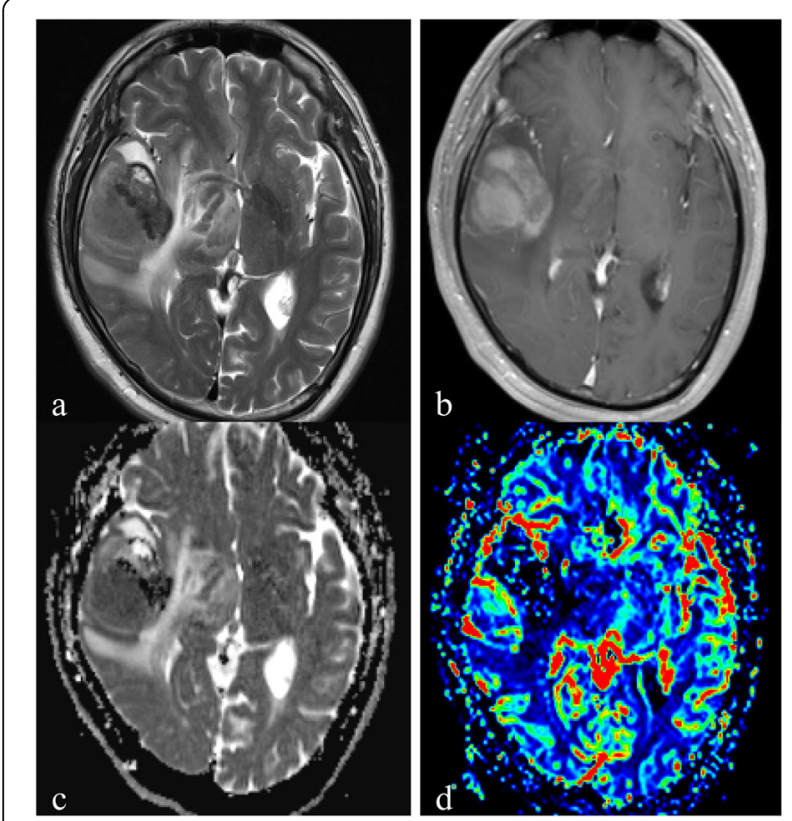

Fig. 2 A 52-year-old man with a primary central nervous system lymphoma in the cortical gray matter. a. Axial T2WI demonstrates the heterogeneous high signal intensity on the right temporal lobe. b. Contrast-enhanced axial T1WI demonstrates a lesion enhancement. c. A corresponding ADC map shows the tumor with a decreased $A D C$ value $\left(A D C_{\text {min }}=0.56 \times 10^{-3} \mathrm{~mm}^{2} / \mathrm{s}\right)$. The $A D C_{\text {mean }}$ value is $0.68 \times 10^{-3} \mathrm{~mm}^{2} / \mathrm{s}$ in the left normal cortical gray matter. $\mathbf{d}$ A correlative color CBV image shows highly elevated perfusion with the calculated $\mathrm{rCBV}_{\max }$ of 3.42

were not significantly different (Fig. 1). In addition, $\mathrm{ADC}_{\text {mean }}$ values of subgroup of the normal control were not significantly different (Fig. 1).

\section{Discussion}

In our study, we present the evidence that $\mathrm{rCBV}_{\max }$ values of PCNSLs are related to their locations. The CGM PCNSLs have the highest $\mathrm{rCBV}_{\max }$, and the WM tumors have the lowest $\mathrm{rCBV}_{\max }$. However, there were no significant differences among $\mathrm{ADC}_{\min }$ values of PCNSLs with different locations.

The role of cMRI in the characterization of PCNSLs has been well established [21]. However, the cMRI features of PCNSLs (homogenous contrast-enhancement pattern and notch sign) at various sites are non-specific.

$\mathrm{ADC}_{\text {min }}$ values have been extensively used to investigate PCNSL and its prognosis [13, 22, 23]. Previous studies have shown a significant inverse correlation between tumor cell density and ADC value in PCNSLs, suggesting ADC to be a surrogate marker for tumor proliferation and Ki-67 index [23-25]. Barajas et al. [11] reported that ADC measurements could predict clinical prognosis in patients with PCNSLs. The study indicated that lower $\mathrm{ADC}_{25 \%}$ and $\mathrm{ADC}_{\text {min }}$ values were predictive of shorter progression-free survival (PFS) and overall

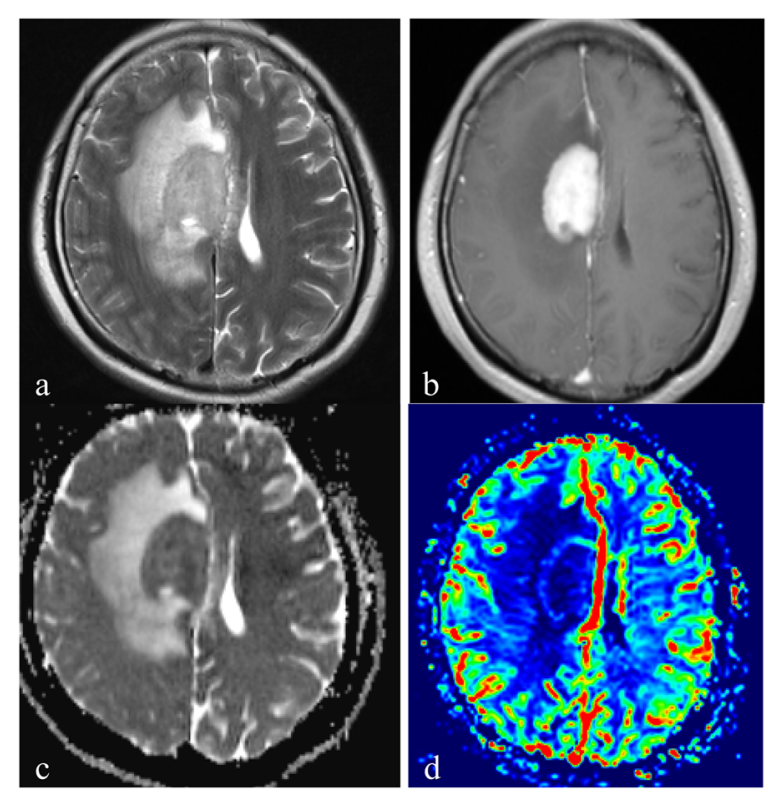

Fig. 3 A 45-year-old woman with PCNSLs in the white matter. a. Axial T2WI shows the homogeneous high signal intensity on the right centrum semiovale. b. Contrast-enhanced axial T1WI demonstrates a lesion enhancement. c. A corresponding ADC map shows the tumor with a decreased $A D C$ value $\left(A D C_{\min }=0.55 \times 10^{-3}\right.$ $\mathrm{mm}^{2} / \mathrm{s}$ ). The $A D C_{\text {mean }}$ value is $0.66 \times 10^{-3} \mathrm{~mm}^{2} / \mathrm{s}$ in the left normal white matter. $\mathbf{d}$. A correlative color CBV image shows slightly elevated perfusion with the calculated $\mathrm{rCBV}_{\max }$ of 0.76

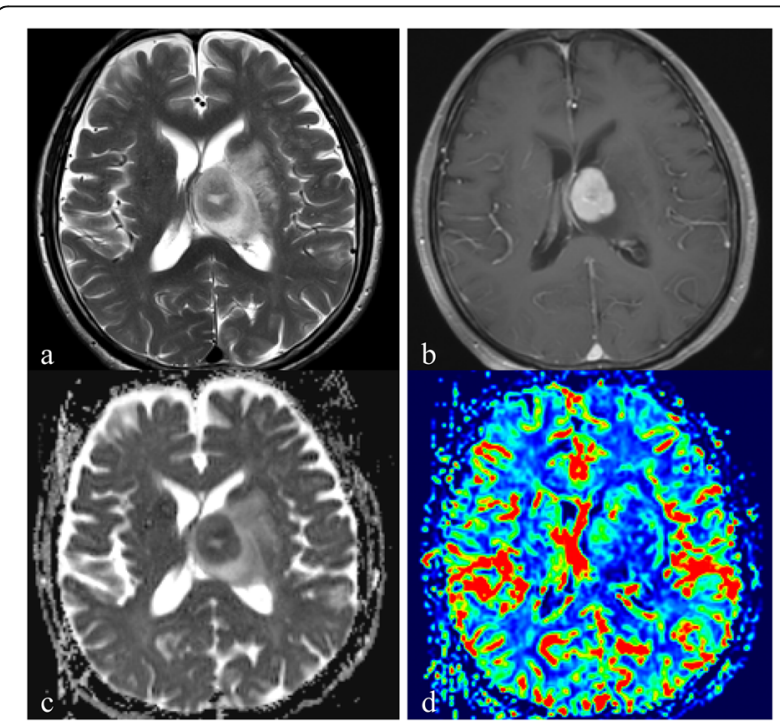

Fig. 4 A 72-year-old man with PCNSLs in the deep gray matter. a. Axial T2WI demonstrates homogeneous high signal intensity on the left basal ganglia region. $\mathbf{b}$. Contrast-enhanced axial T1WI demonstrates a lesion enhancement. c. A corresponding ADC map shows the tumor with a decreased $A D C$ value $\left(A D C_{\min }=0.49 \times 10^{-3}\right.$ $\left.\mathrm{mm}^{2} / \mathrm{s}\right)$. The $A D C_{\text {mean }}$ value is $0.62 \times 10^{-3} \mathrm{~mm}^{2} / \mathrm{s}$ in the right normal deep gray matter. $\mathbf{d}$. A correlative color CBV image shows moderately elevated perfusion with the calculated $\mathrm{rCBV}_{\max }$ of 2.10 
survival (OS). Similarly, Valles et al. [26] found that both mean and minimum ADC values of PCNSLs have strong correlations with patients' PFS and OS. However, as shown by Schob et al., proliferative activity revealed by ADC was not related to the location of PCNSLs [23]. In accordance with the previous study, our research demonstrated no correlations between $\mathrm{ADC}_{\text {min }}$ and the locations of PCNSLs. Meanwhile, our research also confirmed that there was no differences in $\mathrm{ADC}_{\text {mean }}$ values between different locations of control group. Therefore, it is conceivable that DWI variables may not be varied among PCNSLs with different locations.

DSC-PWI has the potential to provide hemodynamic information about PCNSLs [27-30]. In healthy subjects, the CGM structures have the highest blood volume, followed by DGM and WM [31]. PCNSLs represent unique angiocentric growth patterns, but lack abundant neoangiogenesis $[1,32,33]$. Thus, we can infer that the PCNSLs of CGM have the highest rCBV, followed by DGM and WM tumors. Our findings are in good agreement with such a theoretical hypothesis. PCNSLs are usually treated by high-dose methotrexate or radiation therapy [5, 34]. The response to initial methotrexate therapy is significantly associated with patient's prognosis [35]. Clinicians could adjust the drug dose and initiate individualized second-line salvage therapies in chemotherapy-resistant patients, which may minimize neurological toxicity and improve prognosis [36]. It is commonly acknowledged that the delivery of the drug through the vascular system determines the response to chemotherapy [6]. Notably, Valles et al. [26] reported that $\mathrm{rCBV}$ value could be a valid imaging biomarker of clinical outcome: patients of PCNSLs with lower tumor rCBV values at pre-therapy baseline have significantly shorter PFS and OS compared with the control group. Therefore, we can also postulate that PCNSLs of CGM have the best chemotherapeutic response and clinical prognosis, followed by DWM and WM tumors. Location of PCNSLs could stratifies the patients into different risk groups to guide the methotrexate-based chemotherapy.

There are some potential limitations in our study. First, we did not verify the clinical prognostic factors of PCNSLs, although Valles et al. [26] confirmed that tumor rCBV values might be biomarkers of treatment response. A multicentered prospective investigation with long-term follow-up should be further studied to verify our speculation. Second, this retrospective study may lead to some biases in the case selection. Therefore, more extensive trials are needed to validate and establish expected datasets to refine current scores and establish new risk factors. Third, due to the lack of available tumor-tissue samples, we were unable to establish a point to point $\mathrm{rCBV}_{\max }$ measurements associated with histopathological characteristics.

\section{Conclusion}

Our study confirms that the cerebral blood supplies of the PCNSLs are related to their locations. We present the first evidence of tumor location as a simple but effective prognostic indicator of PCNSLs. The PCNSLs of CGM may have the best prognosis, whereas tumors of WM may have the worst prognosis.

\section{Abbreviations \\ MRI: Magnetic resonance imaging; PCNSLs: Primary central nervous system lymphomas; rCBV $_{\text {max }}$ : Relative maximum cerebral blood volume; rCBV: Relative cerebral blood volume; CBV: Cerebral blood volume; $A D C_{\text {min }}$ : Minimum apparent diffusion coefficients; DWI: Diffusion weighted imaging; $A D C_{\text {mean: }}$ Mean apparent diffusion coefficients; $A D C$ : Apparent diffusion coefficients; DSC-PWI: Dynamic susceptibility contrast perfusion- weighted imaging; CMRI: Conventional magnetic resonance imaging; T1WI: T1 weighted imaging; T2WI: T2 weighted imaging; FLAIR: Fluid attenuated inversion recovery; TR: Repetition time; TE: Echo time; TI: Inversion time; CE-T1Wl: Contrast-enhanced T1 weighted imaging; Gd- BOPTA: Gadobente dimeglumine; FOV: Field of view; NEX: Number of excitation; ROI: Region of interest; CGM: Cortical gray matter; WM: White matter; DGM: Deep gray matter; SD: Standard deviation; LSD: Least significant difference; OS: Overall survival; PFS: Progression-free survival}

\section{Acknowledgements}

Not applicable.

\begin{abstract}
Authors' contributions
Z.X. analyzed and interpreted the patients' data regarding Primary central nervous system lymphomas and was a major contributor in writing the manuscript. N.K. participated in the design of the study, performed the statistical analysis. D.C. participated in the design of the study, performed the experiments and the statistical analysis. Y.L. participated in the design of the study and assisted with manuscript preparation. X.Z. participated in the design of the study. Z.X. assisted with manuscript preparation. All authors have read and approved the final manuscript.
\end{abstract}

\section{Funding}

This study was funded by the funding project of medical innovation of Fujian Province (No. 2019-CX-23) and the joint project of health and education of Fujian Province (No. 2019-WJ-10). These funders provide necessary meterials such as ear plugs in the acquisition of MR images and possible fees for open access publication.

\section{Availability of data and materials}

Due to statutory provisions regarding data and privacy protection, the dataset supporting the conclusions of this article is available upon individual request directed to the corresponding author.

\section{Ethics approval and consent to participate}

This work has been carried out with ethics approval from the Fujian Medical University. The need for patient consent was waived.

\section{Consent for publication}

Not applicable.

\section{Competing interests}

The authors declare that they have no competing interests.

\section{Author details}

'Department of Radiology, First Affiliated Hospital of Fujian Medical University, 20 Cha-Zhong Road, Fuzhou 350005, Fujian, China. ²Department of Radiology, Zhongshan Hospital Affiliated to Xiamen University, Xiamen 361004, Fujian, China. ${ }^{3}$ Department of Biomedical Sciences, School of Veterinary Medicine, University of Pennsylvania, Philadelphia, PA 19104, USA. 
Received: 16 December 2019 Accepted: 28 May 2020

Published online: 09 June 2020

\section{References}

1. Koeller KK, Smirniotopoulos JG, Jones RV. Primary central nervous system lymphoma: radiologic-pathologic correlation. Radiographics. 1997;17(6): 1497-526.

2. McAllister LD, Doolittle ND, Guastadisegni PE, Kraemer DF, Lacy CA, Crossen JR, Neuwelt EA. Cognitive outcomes and long-term follow-up results after enhanced chemotherapy delivery for primary central nervous system lymphoma. Neurosurgery. 2000;46(1):51-60 discussion 60-51.

3. Momota H, Narita Y, Maeshima AM, Miyakita Y, Shinomiya A, Maruyama T, Muragaki $Y$, Shibui S. Prognostic value of immunohistochemical profile and response to high-dose methotrexate therapy in primary CNS lymphoma. J Neuro-Oncol. 2010;98(3):341-8.

4. Holdhoff M, Ambady P, Abdelaziz A, Sarai G, Bonekamp D, Blakeley J, Grossman SA, Ye X. High-dose methotrexate with or without rituximab in newly diagnosed primary CNS lymphoma. Neurology. 2014;83(3):235-9.

5. Kansara R, Shenkier TN, Connors JM, Sehn LH, Savage KJ, Gerrie AS, Villa D. Rituximab with high-dose methotrexate in primary central nervous system lymphoma. Am J Hematol. 2015;90(12):1149-54.

6. Goel S, Duda DG, Xu L, Munn LL, Boucher Y, Fukumura D, Jain RK Normalization of the vasculature for treatment of cancer and other diseases. Physiol Rev. 2011;91(3):1071-121.

7. Rubenstein JL, Fridlyand J, Shen A, Aldape K, Ginzinger D, Batchelor T, Treseler $\mathrm{P}$, Berger M, McDermott M, Prados M, et al. Gene expression and angiotropism in primary CNS lymphoma. Blood. 2006;107(9):3716-23.

8. Rubenstein $\mathrm{L}$, Shen A, Batchelor TT, Kadoch C, Treseler P, Shuman MA. Differential gene expression in central nervous system lymphoma. Blood. 2009;113(1):266-7 author reply 267-268.

9. Kang Y, Choi SH, Kim YJ, Kim KG, Sohn CH, Kim JH, Yun TJ, Chang KH. Gliomas: histogram analysis of apparent diffusion coefficient maps with standard- or high-b-value diffusion-weighted MR imaging--correlation with tumor grade. Radiology. 2011;261(3):882-90

10. Yan $R$, Haopeng $P$, Xiaoyuan F, Jinsong W, Jiawen Z, Chengjun $Y$, Tianming Q, Ji X, Mao S, Yueyue D, et al. Non-Gaussian diffusion MR imaging of glioma: comparisons of multiple diffusion parameters and correlation with histologic grade and MIB-1 (Ki-67 labeling) index. Neuroradiology. 2016; 58(2):121-32.

11. Barajas RF Jr, Rubenstein JL, Chang JS, Hwang J, Cha S. Diffusion-weighted MR imaging derived apparent diffusion coefficient is predictive of clinical outcome in primary central nervous system lymphoma. AJNR Am J Neuroradiol. 2010;31(1):60-6.

12. Zhang $Y$, Zhang $Q$, Wang $X X$, Deng $X F$, Zhu $Y Z$. Value of pretherapeutic $D W l$ in evaluating prognosis and therapeutic effect in immunocompetent patients with primary central nervous system lymphoma given high-dose methotrexate-based chemotherapy: ADC-based assessment. Clin Radiol. 2016;71(10):1018-29.

13. Huang WY, Wen JB, Wu G, Yin B, Li JJ, Geng DY. Diffusion-weighted imaging for predicting and monitoring primary central nervous system lymphoma treatment response. AJNR Am J Neuroradiol. 2016;37(11):2010-8.

14. Nagesh V, Chenevert TL, Tsien Cl, Ross BD, Lawrence TS, Junck L, Cao Y. Quantitative characterization of hemodynamic properties and vasculature dysfunction of high-grade gliomas. NMR Biomed. 2007;20(6):566-77.

15. Seeger A, Braun C, Skardelly M, Paulsen F, Schittenhelm J, Ernemann U, Bisdas S. Comparison of three different MR perfusion techniques and MR spectroscopy for multiparametric assessment in distinguishing recurrent high-grade gliomas from stable disease. Acad Radiol. 2013;20(12):1557-65.

16. Barajas RF Jr, Phillips JJ, Vandenberg SR, McDermott MW, Berger MS, Dillon WP, Cha S. Pro-angiogenic cellular and genomic expression patterns within glioblastoma influences dynamic susceptibility weighted perfusion MRI. Clin Radiol. 2015;70(10):1087-95.

17. Cha S. Perfusion MR imaging: basic principles and clinical applications Magn Reson Imaging Clin N Am. 2003;11(3):403-13.

18. Bjornerud A, Sorensen AG, Mouridsen K, Emblem KE. T1- and T2*-dominant extravasation correction in DSC-MRI: part l--theoretical considerations and implications for assessment of tumor hemodynamic properties. J Cereb Blood Flow Metab. 2011;31(10):2041-53.

19. Ginat DT, Mangla R, Yeaney G, Schaefer PW, Wang H. Correlation between dynamic contrast-enhanced perfusion MRI relative cerebral blood volume and vascular endothelial growth factor expression in meningiomas. Acad Radiol. 2012:19(8):986-90.

20. Ho CY, Cardinal JS, Kamer AP, Kralik SF. Relative cerebral blood volume from dynamic susceptibility contrast perfusion in the grading of pediatric primary brain tumors. Neuroradiology. 2015;57(3):299-306.

21. Zhang D, Hu LB, Henning TD, Ravarani EM, Zou LG, Feng XY, Wang WX, Wen L. MRI findings of primary CNS lymphoma in 26 immunocompetent patients. Korean J Radiol. 2010;11(3):269-77.

22. Lu SS, Kim SJ, Kim N, Kim HS, Choi CG, Lim YM. Histogram analysis of apparent diffusion coefficient maps for differentiating primary CNS lymphomas from tumefactive demyelinating lesions. AJR Am J Roentgenol. 2015;204(4):827-34.

23. Schob S, Meyer J, Gawlitza M, Frydrychowicz C, Müller W, Preuss M, Bure L, Quäschling U, Hoffmann KT, Surov A. Diffusion-weighted MRI reflects proliferative activity in primary CNS Iymphoma. PLoS One. 2016;11(8): e0161386.

24. Surov A, Gottschling S, Mawrin C, Prell J, Spielmann RP, Wienke A, Fiedler E. Diffusion-weighted imaging in meningioma: prediction of tumor grade and association with Histopathological parameters. Transl Oncol. 2015;8(6):517-23.

25. Schob S, Meyer HJ, Dieckow J, Pervinder B, Pazaitis N, Höhn AK, Garnov N, Horvath-Rizea D, Hoffmann KT, Surov A. Histogram Analysis of Diffusion Weighted Imaging at 3T is Useful for Prediction of Lymphatic Metastatic Spread, Proliferative Activity, and Cellularity in Thyroid Cancer. Int J Mol Sci. 2017;18(4):821.

26. Valles FE, Perez-Valles CL, Regalado S, Barajas RF, Rubenstein JL, Cha S. Combined diffusion and perfusion MR imaging as biomarkers of prognosis in immunocompetent patients with primary central nervous system lymphoma. AJNR Am J Neuroradiol. 2013;34(1):35-40.

27. Goyal P, Kumar Y, Gupta N, Malhotra A, Gupta S, Gupta S, Mangla M, Mangla R. Usefulness of enhancement-perfusion mismatch in differentiation of CNS lymphomas from other enhancing malignant tumors of the brain. Quant Imaging Med Surg. 2017;7(5):511-9.

28. Nakajima S, Okada T, Yamamoto A, Kanagaki M, Fushimi Y, Okada T, Arakawa Y, Takagi Y, Miyamoto S, Togashi K. Differentiation between primary central nervous system lymphoma and glioblastoma: a comparative study of parameters derived from dynamic susceptibility contrast-enhanced perfusion-weighted MRI. Clin Radiol. 2015;70(12):1393-9.

29. Xing Z, You RX, Li J, Liu Y, Cao DR. Differentiation of primary central nervous system lymphomas from high-grade gliomas by rCBV and percentage of signal intensity recovery derived from dynamic susceptibility-weighted contrast-enhanced perfusion MR imaging. Clin Neuroradiol. 2014;24(4):329-36.

30. Toh CH, Wei KC, Chang CN, Ng SH, Wong HF. Differentiation of primary central nervous system lymphomas and glioblastomas: comparisons of diagnostic performance of dynamic susceptibility contrast-enhanced perfusion MR imaging without and with contrast-leakage correction. AJNR Am J Neuroradiol. 2013;34(6):1145-9.

31. Kuppusamy K, Lin W, Cizek GR, Haacke EM. In vivo regional cerebral blood volume: quantitative assessment with 3D T1-weighted pre- and postcontrast MR imaging. Radiology. 1996;201(1):106-12.

32. Ferreri AJ, Marturano E. Primary CNS lymphoma. Best Pract Res Clin Haematol. 2012;25(1):119-30

33. Kadoch $C$, Treseler $P$, Rubenstein JL. Molecular pathogenesis of primary central nervous system lymphoma. Neurosurg Focus. 2006;21(5):E1.

34. Soussain C, Hoang-Xuan K, Taillandier L, Fourme E, Choquet S, Witz F, Casasnovas O, Dupriez B, Souleau B, Taksin AL, et al. Intensive chemotherapy followed by hematopoietic stem-cell rescue for refractory and recurrent primary CNS and intraocular lymphoma: Societe Francaise de Greffe de Moelle Osseuse-Therapie Cellulaire. J Clin Oncol. 2008;26(15): 2512-8.

35. Chung SR, Choi YJ, Kim HS, Park JE, Shim WH, Kim SJ. Tumor vascular permeability pattern is associated with complete response in Immunocompetent patients with newly diagnosed primary central nervous system lymphoma: retrospective cohort study. Medicine. 2016;95(6):e2624.

36. Jahnke K, Doolittle ND, Muldoon LL, Neuwelt EA. Implications of the bloodbrain barrier in primary central nervous system lymphoma. Neurosurg Focus. 2006:21(5):E11.

\section{Publisher's Note}

Springer Nature remains neutral with regard to jurisdictional claims in published maps and institutional affiliations. 